\title{
LES VALEURS SÉMANTIQUES ET PRAGMATIQUES DES TEMPS VERBAUX DANS LES PROVERBES ZOONIQUES FRANÇAIS ET SERBES
}

\author{
UDC 811.133.1'366.58:82-84 \\ $811.163 .41^{\prime} 366.58: 82-84$
}

\section{Jelena Jaćović, Ivan Jovanović}

Université de Niš, Faculté de philosophie, Département de langue et littérature françaises, Niš, Serbie

\begin{abstract}
Résumé. L'objet de cet article est de déterminer la fréquence et les spécificités des valeurs sémantiques et pragmatiques des temps verbaux passés dans un corpus des proverbes français et serbes. Le corpus est tiré des dictionnaires de référence parémiologique français et serbes (Montreynaud et al. 2006; Maloux 2006; Vigerie 2004; Karadžić 1985 ; Jovanović 2006) et vise les proverbes zooniques. Vu que dans un corpus des proverbes le présent de l'indicatif et la valeur gnomique sont les plus fréquents, notre attention porte particulièrement sur les valeurs des temps passés qui ne sont pas gnomiques. L'analyse sera menée dans le cadre des grammaires sémantiques: "Grammaire du sens et de l'expression » de P. Charaudeau (1992) et « Sintaksa savremenog srpskog jezika-prosta rečenica » de P. Piper et al. (2005).
\end{abstract}

Mots-clés : sémantique des temps verbaux, français, serbe, proverbes zooniques, analyse contrastive.

\section{INTRODUCTION}

Jusqu'aux années soixante-dix du $\mathrm{XX}^{\mathrm{e}}$ siècle les proverbes étaient l'objet d'un intérêt folklorique ou thématique, ainsi que l'objet d'études littéraires, comme par exemple ceux chez Cervantès ou Balzac. D'après les auteurs tels que Jordana Ferary (Ferary 2017, 5), Jean-Claude Anscombre (Anscombre 2012, 21), Visetti et Cadiot (Visetti, Cadiot 2006, 8 ), les grammaires traitent les proverbes en tant qu'une sorte de locution à laquelle on n'accorde pas beaucoup d'attention puisqu'ils sont considérés en tant que formes archaïques. Néanmoins, depuis une trentaine d'années les linguistes travaillent dans le

Submitted July 1, 2019; Accepted September 19, 2019

Corresponding author: Ivan Jovanović

University of Niš, Faculty of Philosophy

E-mail: ivan.jovanovic@filfak.ni.ac.rs 
cadre du champ proverbial en examinant les proverbes du point de vue sémantique et syntaxique. Les pionniers qui ont abordé le phénomène des proverbes étaient Georges Kleiber et Jean Claude-Anscombre et ont été succédés par de nombreux spécialistes ayant consacré leurs recherches aux aspects syntaxique, sémantique et pragmatique des proverbes. Il s'agit des études novatrices qui se servent de cadres linguistiques pour l'analyse de marqueurs du discours, des temps verbaux, de la prosodie, du rythme, de la rhétorique etc. Parmi les sujets abordés dans les études linguistiques portant sur les proverbes, la généricité, les stéréotypes ou la métaphore ont fait l'objet de nombreux travaux (Anscombre 2000, 6-26 ; Kleiber 1999, 515-531 ; Tamba 2000, 110-118). Taylor $(1931,36)$ a souligné que le proverbe est une question épineuse et délicate qui n'est pas encore résolue et il reste toujours des aspects à étudier pour parvenir à sa circonscription. Ainsi, en terme des recherches et des études contrastives proverbiales en français et en serbe (Gudurić, Drobnjak 2012, 56-64 ; Stanković, Jovanović 2013, 363-374 ; Jovanović, Stanković 2014, 383-397 ; Jovanović 2014a, 487-503 ; 2014b, 643-651 ; 2017, 203-213 ; 2018, 587-600 ; 2019 ; Jovanović, Jaćović 2016, 77-95), il est à remarquer qu'elles touchent aux aspects lexico-sémantique, linguistique, culturel et syntaxique de ces unités appartenant aux divers champs thématiques (temps, espace, animaux, religion, famille, relation humaine, corps humain) et qu'aucune recherche n'a été consacrée aux valeurs éventuelles des temps verbaux en dehors de la valeur gnomique.

Quant à la définition du proverbe, il est considéré comme une forme figée, c'est-àdire comme une unité polylexicale codée, possédant à la fois une certaine rigidité ou fixité de forme et une certaine fixité référentielle ou stabilité sémantique qui se traduit par un sens préconstruit, c'est-à-dire par convention pour tout locuteur, qui fait donc partie du code linguistique commun (Kleiber 2000, 40 ; Visetti, Cadiot 2006, 69). D. MrševićRadović remarque que le proverbe joue un rôle indispensable sous l'angle de la tradition et de la culture des peuples en s'inscrivant dans le patrimoine langagier et culturel et en touchant à tous les domaines de la vie de l'homme (Mršević-Radović 2008, 2). Donc, les proverbes sont des témoins irréfutables d'une certaine époque qui diffère de celle à laquelle nous appartenons. Pour de nombreux locuteurs d'une communauté langagière les proverbes représentent un grand inconnu et de ce fait méritent d'être conservés et étudiés afin de saisir des valeurs et des traditions des époques antérieures dans les sociétés différentes. Ceci également permet de compléter une image langagière du monde (Muñoz 2000, 91 ; Jovanović 2018, 588).

Le présent article vise à examiner les valeurs des temps verbaux passés dans les proverbes français et serbes en tâchant de déterminer surtout l'existence des valeurs qui ne sont pas gnomiques. Le corpus est tiré des dictionnaires de référence proverbiaux français et serbes et vise les proverbes zooniques parce qu'il s'agit d'un domaine riche et varié dans lequel nous avons identifié un grand nombre d'exemples : P. Vigerie 2004 : Quand on parle du loup, on en voit la queue, M. Maloux 2006 : Dictionnaire des proverbes, sentences et maximes, F. Montreynaud et al. 2006 : Dictionnaire de proverbes et dictons, V. Karadžić 1985 : Srpske narodne poslovice, J. Jovanović 2006 : Knjiga srpskih narodnih poslovica. Vu le fait que dans un corpus proverbial la valeur gnomique des temps verbaux est la plus fréquente et incontournable, on s'attache à explorer d'autres valeurs en se limitant exclusivement aux temps verbaux du passé. L'analyse sera menée dans le cadre des grammaires sémantiques : "Grammaire du sens et de l'expression » (1992) de Patrick Charaudeau pour le corpus français et «Sintaksa savremenog srpskog jezika - prosta rečenica » (2005) de Predrag Piper et al. pour le corpus serbe. 


\section{CADRE THEORIQUE ET METHODOLOGIQUE}

Malgré un bon niveau de ressemblances en termes de caractéristiques formelles et catégoriales des systèmes verbaux français et serbe, il existe aussi des différences cruciales surtout dans le domaine d'aspect, de mode, de concordance des temps et de nombre des temps verbaux dans la langue standard. L'aspect est en serbe une catégorie morphologique qui synthétise au niveau lexical les informations aspectuelles : l'aspect perfectif ou imperfectif du lexème verbal est determiné par les préfixes. Le serbe dispose d'un nombre restreint des formes verbales par rapport au français et la concordance des temps est un phénomène dont à l'origine se trouve le fait que le "point de référence » ${ }^{1}$ est positionné au passé. Dans la langue serbe ce phénomène n'existe pas, parce que pour exprimer la simultanéité, postériorité ou antériorité par à rapport à tous les points de référence possibles, on utilise les mêmes formes verbales (le présent, le parfait et le futur I). Le nombre réduit des temps verbaux en serbe est la conséquence d'un changement dans l'expression de l'aspect - l'imparfait serbe n'est plus utilisé et sa place est prise par le parfait des verbes inachevés. L'aoriste en serbe est limité à l'usage interprétatif, alors que les valeurs du passé simple en français sont exprimées par le présent perfectif. L'usage du plus-que-parfait serbe est réduit à l'interprétation résultative, dont le résultat suppose une action antérieure achevée, alors qu'en français il existe aussi l'interprétation de l'antériorité. Certaines formes verbales n'existent pas en serbe (le parfait antérieur) ou elles ne sont pas très fréquentes (le conditionnel passé) et la plus grande correspondance des formes se trouve parmi les formes exprimant le futur (Ašić, Stanojević 2008, 185).

Les études contrastives franco-serbes menées dans le domaine des temps verbaux sont très nombreuses et variées : sur l'aspect (Drašković 1969, Točanac 1988, Gudurić 1991, Vilić 2016), sur la transitivité (Gudurić 2006), sur l'analyse des valeurs sémantico-pragmatiques des formes verbales (Drašković 1969, Petrović 1991, Točanac 1991, Petrović 2002, Ašić, Stanojević 2008, Gudurić 2011, Jovanović 2013, Dodig 2019), pour ne pas mentionner que quelques-unes. Pourtant, ces études n'étaient jamais réalisées sur un corpus parémiologique. D'ailleurs, les slavistes français ont montré un certain intérêt pour les études des proverbes serbes (Thomas 2012).

Pour démontrer les valeurs sémantiques des temps du passé dans les proverbes françaises et serbes nous avons choisi de suivre la systématisation de deux grammaires d'orientation sémantique. Ce choix est justifié par le fait que les deux grammaires s'appuient considérablement sur les acquis de la sémantique cognitive et organisent leurs systématisations autour des catégories sémantiques (modalité, télicité, temporalité, etc.). D'après les grammaires traditionnelles, « le temps verbal » est en effet une catégorie formelle censée d'avoir une certaine valeur temporelle, alors que Charaudeau estime que le temps est le résultat d'une construction - représentation du monde qui se compose de plusieurs aspects ou visions (Charaudeau 1992, 456). Tous les processus peuvent être considérés selon différents points de vue qu'il nomme « visions » et la « situation dans le temps » en est un. Elle donne la position par rapport à la référence de l'acte d'énonciation et engage les catégories conceptuelles de coïncidence, antériorité et postériorité (Charaudeau 1992, 452).

Les formes verbales peuvent avoir des valeurs différentes selon le système d'énonciation où elles sont employées, ce qui a été constaté par Benveniste (1976). Ce

\footnotetext{
${ }^{1}$ La notion de «point de référence » a été introduite par le logicien H. Reichenbach (Elements of Symbolic Logic 1947), étant donné que les deux points existants («point de parole » et «point d'évènement ») se sont montrés insuffisants pour la description de tous les usages des temps verbaux.
} 
système d'énonciation est formulé d'abord comme la distinction entre «histoire » et « discours» et puis défini par Weinrich (1985) en tant que « récit» et «commentaire». Certaines recherches ont montré que les proverbes, selon ce critère, ne peuvent être rangés que dans un système d'énonciation spécifique (Thomas 2012, 575). La spécificité du système de l'énonciation des proverbes est accentuée par la référence temporelle qui se trouve à l'intérieur des proverbes mêmes et ne sort pas du contexte phrastique. Puisque le présent de l'indicatif et les formes impersonnelles sont les plus présents dans les parémies françaises et serbes, notre attention porte, cette fois-ci, sur les valeurs non-gnomiques des formes verbales du passé françaises (passé composé, passé simple, imparfait) et serbes (parfait, aoriste).

\section{ANALYSE ET RESULTATS}

Dans la suite nous allons présenter les résultats de notre recherche en comparant les valeurs retrouvées dans les corpus français et serbe. Dans le corpus français figurent les temps verbaux suivants : le passé composé, le passé simple et l'imparfait. Le corpus serbe ne dispose pas d'un grand nombre des temps verbaux - on n'y trouve que le parfait et l'aoriste. Il est important à constater que le plus-que-parfait n'existe pas du tout dans le corpus et que l'on rencontre l'imparfait seulement dans le corpus français.

\subsection{Le passé composé}

Le passé composé est le temps verbal français le plus exploité pour exprimer les processus dans le passé. Il est capable d'exprimer deux valeurs fondamentales : celle d'un «passé révolu » et d'un " présent accompli ». La valeur d'un "passé révolu » exprime un processus réalisé entièrement et coupé du présent, alors que la valeur du « présent accompli » démontre un processus déjà au passé qui garde, pourtant, un contact avec le présent actuel. Dans notre corpus, le passé composé était la forme la plus employée parmi les temps du passé.

\subsubsection{Valeur de généralité}

Le contact maintenu avec le présent, à travers la valeur du «présent accompli », lui procure la possibilité d'une interprétation générique, d'où sa valeur de généralité. Cette valeur lui fournit une place à côté du présent générique ce qui le rend apte à se retrouver dans les proverbes La valeur de généralité s'est montrée très fréquente, étant donné que le passé composé d'une valeur gnomique peut être remplacé par le présent, puisque les deux formes expriment la valeur omnitemporelle :

(1) Jamais petit chat n'a porté rat à sa mère.

(2) On n'a jamais vu une chèvre morte de faim.

Dans les exemples précédents la valeur de généralité du passé composé est mis en relief par les adverbes de temps «jamais » et, dans l'exemple qui suit, elle est exprimée par article défini :

(3) La chèvre a pris le loup. 


\subsubsection{Valeur d'un « présent accompli »}

La valeur qui distingue le passé composé des autres temps du passé du système verbaux français est notamment celle qui produit un effet de contact entre situation passée et présente la valeur d'un « présent accompli ». En outre, Charaudeau souligne que cette valeur du passé composé permet un décrochage interprétatif - le narrateur fait un commentaire sur le passé qui a encore une répercussion dans le présent (Charaudeau 1992, 469). C'est un procédé qui paraît convenable pour le contexte proverbial puisqu'elle permet de rendre plus vif un proverbe à chaque emploi. Par conséquent, cette valeur est assez fréquente dans notre corpus et exprime une sorte de cause dont les conséquences se trouvent au présent :

(4) Voilà ce que les rats n'ont pas mangé

(5) Quand le rossignol a vu ses petits, il ne chante plus.

Il y a des cas où cette conséquence n'est pas exprimée et la phrase reste simple exprimant une sorte de cause dont les conséquences sont présentes dans la situation actuelle :

(6) Nous n'avons pas gardé les cochons/les dindons/les oies ensemble.

(7) Le rat a mis le museau dans la poix.

Il y a des cas où le passé composé avec la valeur du «présent accompli » dans la proposition subordonnée circonstancielle affiche l'antériorité par rapport à un avenir hypothétique, exprimé par le conditionnel, et au point de référence non-exprimé ancré dans le moment de l'énonciation :

(8) On ne saurait retenir le chat quand il a goûté la crème.

\subsubsection{Valeur d'un « passé révolu»}

Sa valeur primordiale est de signaler un « passé révolu », mais dans un cadre proverbial, la valeur temporelle n'est pas la plus importante - l'accent est mis sur l'effet de l'aspect accompli et c'est l'idée d'un processus déjà réalisé se positionnant hors contexte temporel qui est mise en avant :

\section{(9) Où la guêpe a passé, le moucheron demeure.}

La valeur de la ponctualité d'un processus passé permet aussi de créer une suite d'actions qui se succèdent. De plus, l'accumulation de cet emploi crée un effet d'isolement des faits les uns par rapport aux autres et donne un aspect insulaire (Charaudeau 1992, 469) ce qui lui permet d'assumer un rôle dans les proverbes exprimant des actions qui se succèdent :

(10) Quand Jean bête est mort, il a laissé bien des héritiers.

(11) Il a battu les buissons et un autre a pris les oiseaux.

(12) L'un a battu les buissons, l'autre a pris les oisillons. 
Parfois, le passé composé à valeur de «passé révolu » exprime, dans le cadre d'une phrase complexe, l'antériorité par rapport à un processus qui se trouve au présent et pour lequel la phrase simple dont le noyau verbal se trouve au passé composé avec cette valeur représente la cause :

(13) Il entend le jars : il a mené les oies.

(14) Il est trop tard pour fermer l'écurie quand le cheval s'est sauvé.

\subsection{Le parfait}

Le parfait en serbe a le même positionnement syntaxique dans le système des temps verbaux que son homologue français le passé composé. Il exprime un processus au passé sans donner plus de précision, ce qui lui permet l'usage le plus large pour exprimer un processus du passé en serbe. Dans le corpus parémiologique on trouve majoritairement sa forme tronquée (krnji perfekat - le parfait tronqué), qui suggère déjà un usage spécifique, ainsi que quelques usages atemporels.

\subsubsection{Le parfait tronqué - valeur expressive}

Cette forme est chargée d'expressivité et stylistiquement condensée. Son usage est fréquent dans le style fonctionnel journalistique pour marquer l'inattendu d'un événement. Dans les proverbes le parfait tronqué apparait avec les verbes d'aspect perfectif et exprime ainsi la valeur générale à travers un exemple isolé. Quand il s'agit des verbes imperfectifs, ils donnent une signification itérative (Piper et al. 2005, 402). Dans notre corpus les exemples avec parfait tronqué sont les plus fréquents :

(15) Pristao deda među goveda.

(16) Potukle se zebe oko tuđeg prosa.

(17) Vid'la žaba da se konji kuju pa i ona digla nogu.

(18) Bežao od vaške pa pao medvedu u šake.

\subsubsection{Valeur d'un acte réalisé au passé}

La valeur de base du parfait serbe est d'exprimer un acte réalisé au passé ${ }^{2}$. Cette valeur peut être manifestée par les verbes imperfectifs et dans ce cas, elle marque la répétition :

(19) Koga su zmije klale i guštera se boji.

(20) Čaplja je svaku pticu učila da pliva, a sama se udavila.

ou une durée indéterminée :

(21) I mačka je kralja gledala, ali ga se nije bojala.

\footnotetext{
${ }^{2}$ Dans la grammaire serbe, il existe la distinction entre deux types de détermination temporelle. D'un côté, c'est l'indicatif (la détermination indicative) où le processus est positionné par rapport au moment de la parole et, de l'autre côté, c'est le relatif (la détermination relative) où le processus se positionne par rapport à un autre processus (Piper et al. 2005, 346).
} 
Lorsque la forme perfective du verbe au parfait ${ }^{3}$ désigne un résultat qui atteint le présent, il est orienté vers le moment de l'énonciation :

(22) Još se nije okozila, a kozle igra po polju.

(23) Rugala se sova senici: idi kučko glavata.

(24) Kud je otišlo june, nek ide i uže.

Quand il y a une suite des verbes perfectifs au parfait, cela crée une séquence narrative qui rentre dans le domaine de l'énonciation de récit (Thomas 2012, 565):

(25) Dok su medveda navukli na kruške, otrgli mu uši; a dokle su ga svukli s krušaka otrgli mu rep.

\subsubsection{Valeurs modales}

Parmi les valeurs modales, la plus fréquente dans notre corpus est celle exprimant l'hypothèse - dans la subordonnée conditionnelle, le parfait serbe exprime la condition passée réelle située dans un contexte gnomique :

(26) Ako je Bog uzeo konju noge, nije njisak.

(27) Ako je konju poginuo trk, nije prdnjava.

(28) Ako je pas pitu izeo, tepsija je ostala.

On trouve ainsi la valeur de la certitude avec une nuance de réprimande, toujours dans les phrases à la négation :

(29) Nije ti krava na nogu stala.

(30) Nisam s tobom svinje čuvao.

(31) Nije mu vrana mozak popila.

\subsection{Le passé simple}

Son positionnement dans le système verbal français lui attribue la valeur d'un « passé révolu » où le processus est complétement détaché du « présent actuel ». Ce processus est antérieur au moment de l'acte de l'énonciation et il est perçu comme ponctuel.

L'emploi du passé simple crée un effet de la distanciation historique qui est utilisée dans les récits et devient la marque du genre littéraire (Charaudeau 1992, 467). L'éloignement par rapport à l'actualité du narrateur le fait susceptible d'être exploitée dans les proverbes et de créer le cadre gnomique :

(32) Ce qui ne fut jamais ni ne sera, c'est le nid d'une souris dans l'oreille d'un chat.

(33) Pour un point, Martin perdit son âne.

Dans notre corpus, les exemples avec ladite valeur sont parfois en convergence avec les adverbes de temps tels que jamais qui est suivi d'un nom sans déterminant :

\footnotetext{
${ }^{3}$ Il s'agit souvent des verbes pronominaux (Thomas 2012, 564).

${ }^{4}$ Dans cet exemple il y a une alternance des formes de parfait et de parfait tronqué.
} 
(34) Jamais chat emmitouflé ne prit souris.

(35) Jamais cheval ni méchant homme n'amenda pour aller à Rome.

(36) Jamais un coup de pied de jument ne fit mal à un cheval.

\subsection{L'aoriste}

La valeur de l'aoriste dans la langue serbe standard est double : il exprime des processus d'un passé immédiat, ainsi que des processus d'un passé révolu (complètement achevé et détaché du moment présent). Il a une autre caractéristique marquante qui le distingue nettement du parfait : il présente le processus comme vécu (Piper et al. 2005, 424).

\subsubsection{Valeur non-référentielle}

La valeur unique de l'aoriste retrouvée dans le corpus est la valeur gnomique. C'est un usage non-référentiel qui exprime « un fait d'expérience général, universel qui est d'habitude manifesté par le présent » (Thomas 2012, 568) :

(37) Mnogo pasa ujedoše vuka.

(38) Izede konja trava.

(39) Ko tolikoj vojsci konje posedla? Svak svoga.

Pourtant, il est possible de dégager plusieurs nuances sémantiques qui s'esquissent dans le cadre de la valeur gnomique. Tout d'abord, il est à noter que les verbes à l'aoriste peuvent être à la première personne ce qui renvoie au locuteur sans renvoyer au moment de l'énonciation et donne une nuance plus expressive :

(40) Ja silom ne ulovih zeca.

Parfois, c'est le nom à la forme vocative assumant la fonction appellative qui aide à l'actualisation du moment de l'énonciation, ainsi que le pronom à valeur stylistique :

(41) Svi se konji zaigraše, deder i ti sivče.

Une suite des verbes à l'aoriste sont en mesure d'établir une séquence narrative « instaurant une expériance édifiante pour le locuteur qui la reprend à son compte » (Thomas 2012, 567) :

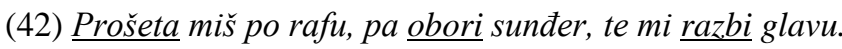

\subsection{L'imparfait}

Cette forme se trouve seulement dans le corpus français, on ne le rencontre pas dans le corpus serbe. L'imparfait français exprime la transposition du «présent actuel » dans un moment qui n'est pas l'actualité du sujet parlant et cette position évoque deux valeurs fondamentales : celle de «l'actualité dans le passé » qui produit un effet de permanence et celle du «passé, présent ou futur imaginés » exploitée dans l'expression de l'hypothèse ce qui est la seule valeur qui se trouve dans notre corpus. 


\subsubsection{Valeur d'hypothèse}

La valeur prédominante de l'imparfait est celle exprimant l'hypothèse dans le présent car tous les proverbes à l'imparfait de notre corpus sont donnés sous forme de phrases conditionnelles :

(43) Si le ciel tombait, il y aurait bien des alouettes de prises.

(44) Si on savait où le loup passe, on irait l'attendre au trou.

(45) Si tous les fous portaient un bonnet blanc, nous ressemblerions à un troupeau d'oies.

\section{CONCLUSIONS}

En guise de conclusion, nous pouvons constater que le champ proverbial analysé repose sur trois temps verbaux passés: passé composé (parfait serbe), passé simple (aoriste serbe) et imparfait qui, en fonction $\mathrm{du}$ contexte, se ressemblent ou se différencient sur le plan sémantique. En ce qui concerne le passé composé, la valeur la plus fréquente et celle de généralité et puis celle d'acte accompli au présent et au passé. Une telle fréquence réside dans le fait que les différents adverbes de temps et les articles définis renforcent cette généralité visant à faire dégager les vérités générales et les règlements sur le monde et son entourage. À la différence du passé composé, dans la langue serbe c'est le parfait tronqué à valeur expressive qui prédomine les autres valeurs telles que celle d'acte accompli au passé ou la valeur modale. Quant au passé simple, il ne possède que la valeur du passé révolu sous-entendant le procès complètement détaché du moment de la parole alors que l'aoriste serbe se caractérise par la valeur gnomique. Ces deux temps sont utilisés dans les proverbes pour insister sur leur ponctualité. L'imparfait français est en usage dans les proverbes pour se référer à une hypothèse. Les proverbes sous forme de phrases hypothétiques représentent un champ fécond pour exprimer le contenu gnomique. Dans la langue serbe, par rapport à la langue française, l'imparfait n'est identifié en aucun exemple car il est sorti de l'usage en seconde moitié du XIX ${ }^{\text {ème }}$ siècle et est substitué par le parfait. Il est à souligner que le plus-que-parfait ne figure pas dans notre corpus car il peut être remplacé par le passé simple pour placer le procès dans une durée sur l'axe temporel et c'est certainement une des raisons pourquoi il est abandonné dans les proverbes.

Note : Cet article est rédigé dans le cadre du projet scientifique Les langues, les littératures et les cultures romanes et slaves en contact et en divergence ( $n^{\circ}$ 81/1-17-8-01) soutenu par la Faculté de philosophie de l'Université de Niš, l'AUF (Agence universitaire de la Francophonie) et l'Ambassade de France en Serbie.

\section{RÉFÉRENCES}

ANSCOMBRE, Jean-Claude. «Pour une théorie linguistique du phénomène parémique », dans La parole exemplaire. Introduction à une étude linguistique des proverbes. Paris : Armand Colin, 2012, p. 21-39.

ANSCOMBRE, Jean-Claude. «Proverbes et formes proverbiales : valeur évidentielle et argumentative ». Langue française, 1994, 102, p. 95-107.

ANSCOMBRE, Jean-Claude. « Parole proverbiale et structure métrique ». Langage, 2000, 139. P. 6-26.

ASIC, Tijana, et Veran STANOJEVIC. Semantika i pragmatika glagolskih vremena u francuskom i srpskom jeziku. Kragujevac, Filološko-umetnički fakultet, 2008. 
BenVEniste, Émile. Problèmes de linguistique générale, tome 1. Paris, Gallimard, 1976.

Charaudeau, Patrick. Grammaire du sens et de l'expression. Paris, Hachette, 1992.

DRASKOVIC, Vlado. Ogledi iz sintakse $i$ istorije francuskog jezika. Beograd, Filološki fakultet Univerziteta u Beogradu, 1969.

DoDIG, Milana. «Le conditionnel dans la langue française et ses équivalents sémantiques dans la langue serbe : étude comparatvie entre le conditionnel français et le potentiel serbe ». Thèse de doctorat. Université de Montpelier, 2019. https://tel.archives-ouvertes.fr/tel-02058884

DRoBNJAK, Dragana, GUDURIC, Snežana, et Ana TOPOLJSKA. «O starosti u francuskom, srpskom i slovačkom poslovičnom izražavanju », dans Jezici i kulture u vremenu i prostoru I. Novi Sad, Filozofski fakultet, $2012,55-64$

FERARY, Sonia Gomez-Jordana. « Le proverbe. Forme, sens et rythme ». Scolia, 31/2017, 2017, p. 5-17.

GUDURIC, Snežana. «Neke aspektualne osobenosti srpskohrvatskog i francuskog infinitiva », dans Zbornik ISJK, 10. Novi Sad: Filozofski fakultet, 1991, 71-75.

GUDURIC, Snežana. «O tranzitivnosti - iz ugla romanske lingvistike ». U Godišnjak Filozofskog fakulteta u Novom Sadu, XXXI, 2006, p. 165-180.

GuDURIC, Snežana. «L'expression de l'hypothèse en français et en serbe : un aperçu général ». Nasleđe, 19 /VIII, 2011, p. 235-246.

JovANOVIC, Jelena. Knjiga srpskih narodnih poslovica, knjiga 1. Beograd, Naučno društvo za negovanje i očuvanje srpskog jezika, 2006.

Jovanovic, Vera. "Perfekat u srpskom jeziku i njegovi ekvivalenti u francuskom jeziku ». Doktorska disertacija, Univerzitet u Kragujevcu, 2013. https://fedorakg.kg.ac.rs/fedora/get/o:682/bdef:Content/get

Jovanovic, Ivan, Stankovic, Selena. "Sur quelques pronoms dans les proverbes français et sur leurs équivalents / correspondants serbes ». Philologia Mediana 6, 2014, p. 383 - 397.

JovANOVIC, Ivan. «Semantički konstituenti lekseme chèvre - koza u francuskim poslovicama i njihovim srpskim ekvivalentima: primer marginalizacije prototipičnih osobina », dans Jezik, književnost, marginalizacija, Jezička istraživanja. Niš, Filozofski fakultet, 2014, p. 487-503.

JovANOVIC, Ivan. «Francuske poslovice s leksemom Dieu - Bog i njihovi srpski ekvivalenti ». Crkvene studije 11,2014 , p. 643-651.

JOVANOVIC, Ivan, JACOVIC, Jelena. « Sintaksičke odlike poslovica u francuskom jeziku u poređenju sa srpskim jezikom », dans Primenjena lingvistika danas, Zbornik radova u čast Dušanki Točanac. Beograd: Društvo za primenjenu lingvistiku, 2016, p. 77-95.

JOVANOVIC, Ivan. 2017. „Francuske i srpske paremije u leksičko-semantičkom polju vreme“. U Jezik, književnost, vreme. 203 - 213. Niš: Filozofski fakultet.

JOVANOVIC, Ivan. «Francuske i srpske paremije u leksičko-semantičkom polju prostor », dans Jezik, književnost, prostor. Niš, Filozofski fakultet, 2018, p. 587-600.

KARADZIC, Vuk. Srpske Narodne poslovice. Beograd, Prosveta-Nolit, 1985.

KLEIBER, Georges. La Sémantique du prototype. Paris, PUF,1994.

KLEIBER, Georges. «Proverbes : sens et dénomination ». Nouveaux cahiers d'allemend, 17-3, 1999, p. 515531.

KLEIBER, Georges. « Sur le sens ses proverbes ». Langages, 139, 2000, 39-58.

MalouX, Morice. Dictionnaire des proverbes, sentences et maximes. Paris, Larousse, 2006.

MonTREYNAUd, Florence et al. Dictionnaire de proverbes et dictons. Paris, Le Robert, 2006.

Mrsovic-RAdovic, Dragana. Frazeologija $i$ nacionalna kultura. Beograd, Društvo za srpski jezik i književnost, 2008.

MUÑOZ, Sonia. «Les proverbes et phrases proverbiales français et leurs équivalences en espagnol ». Langages, $34^{\mathrm{e}}$ année, $\mathrm{n}^{\circ} 139,2000$, p. $98-109$.

PETROVIC, Nada. Francuska glagolska vremena I. Komparativna analiza francuskog i srpskohrvatskog pluskvamperfekta. Beograd, Naučna knjiga, 1991.

PETROVIC, Nada. Francuska glagolska vremena II. Imperfekat, aorist, perfekat. Beograd, Filološki fakultet Narodna knjiga, 2002.

PIPER, Predrag, IVANA Antonić, Vladislava RUZIC, Sreto TANASIC, Ljudmila PoPOVIC, et Branko TosoviC. Sintaksa savremenog srpskog jezika: Prosta rečenica. Beograd, Institut za srpski jezik SANU, Beogradska knjiga - Novi Sad, Matica srpska, 2005.

STANKOVIC, Selena, et Ivan JOVANOVIC. «L'emploi des pronoms dans les proverbes français avec les noms d'animaux domestiques et dans leurs équivalents/correspondants en serbe », dans Agapes francophones. Timișoara, Université de Vest, 2013, p. 363-374.

TAMBA, Irène. « Formule et dire proverbial ». Langages, 139, 2000, p. 110-118.

TAYLOR, Archi. The proverb. Harvard University, Press Cambridge - Massachusetts, 1931. 
TOČANAC, Dušanka. « Obeležja glagolskog aspekta u srpskohrvatskom i francuskom jeziku (1) », dans Zbornik radova Instituta za strane jezike i književnosti, 9. Novi Sad, Filozofski fakultet, 1988, p. 83-99,

TOČANAC, Dušanka. « Transpozicija srpskohrvatskog oblika budem+particip u francuski sintaksički sistem », dans Zbornik radova sa IV simpozijuma Kontrastivna jezička proučavanja. Novi Sad, Jugoslovensko društvo za primenjenu lingvistiku, 1991, p. 271-277.

ThomAs, Paul-Louis. « Temps, mode, aspect et système d'énonciation des proverbes BCMS », dans Revue des études slaves, tome 83, fascicule 2-3, La lettre et l'esprit : entre langue et culture. Études à la mémoire de Jean Breuillard, 2012, p. 557-577.

VIGERIE, P. Quand on parle du loup, les animaux dans les expressions françaises. 2. éd. Paris, Larousse, 2004.

VILIC, Ivana. «Teličnost kao distinktivno obeležje aspektualnog značenja u francuskom i srpskom jeziku ». Doktorska disertacija, Univerzitet u Novom Sadu, 2016. https://www.cris.uns.ac.rs/searchDissertations.jsf

VISETTI, Yves-Marie et Pierre CADIOT. Motifs et proverbes. Essai de sémantique proverbiale. Paris, PUF, 2006.

WEINRICH, Harald. Grammaire textuelle du français. Paris, Didier, 1985.

\section{SEMANTIČKE I PRAGMATIČKE VREDNOSTI GLAGOLSKIH VREMENA U FRANCUSKIM I SRPSKIM POSLOVICAMA SA ZOONIMSKOM KOMPONENTOM}

Cilj rada je da se odrede vrste i frekvencije semantičkih i pragmatičkih vrednosti prošlih glagolskih vremena na korpusu francuskih i srpskih poslovica. Korpus je ekscerpiran iz francuskih i srpskih paremioloških rečnika (Montreynaud et al. 2006; Maloux 2006; Vigerie 2004; Karadžić 1985; Jovanović 2006) i obrađuje poslovice sa zoonimskom komponentom. Budući da je u ovakvoj vrsti korpusa prezent najčešći glagolski oblik i gnomska vrednost najprisutnija, naša pažnja je naročito usmerena na ostale vrednosti prošlih vremena i analiza se sprovodi u okviru semantičkih gramatika Patrika Šarodoa „Grammaire du sens et de l'expression“ (1992) i Predraga Pipera i $d r$. ,Sintaksa savremenog srpskog jezika - prosta rečenica“ (2005).

Ključne reči: semantika glagolskih vremena, francuski jezik, srpski jezik, poslovice sa zoonimskom komponentom, kontrastivna analiza 